\title{
Assessment of the effect of nanosilica on the mechanical performance and durability of cementitious materials
}

\author{
Gerlinde Lefever ${ }^{1, \star}$, Dimitrios G. Aggelis ${ }^{1}$, Nele De Belie ${ }^{2}$, Didier Snoeck ${ }^{2}$, and Danny Van Hemelrijck ${ }^{1}$ \\ ${ }^{1}$ Department of Mechanics of Materials and Constructions, Vrije Universiteit Brussel, Pleinlaan 2, 1050 Brussels, Belgium \\ ${ }^{2}$ Magnel Laboratory for Concrete Research, Department of Structural Engineering, Faculty of Engineering and Architecture, \\ Ghent University, Tech Lane Ghent Science Park, Campus A, Technologiepark Zwijnaarde 904, B-9052 Gent, Belgium
}

\begin{abstract}
Over the last years, nanotechnology is getting more attractive and nanomaterials are being used more commonly in construction industry. One of these materials is nanosilica: the nano-sized, engineered form of silica fume. The replacement of cement by these nanoparticles is said to enhance both the mechanical performance and the durability of the concrete material. In this paper colloidal silica will be used, which is nanosilica in solution. A characterization of mortar mixtures containing different amounts of silica is done and a comparison is made with respect to a reference mixture.
\end{abstract}

\section{Introduction}

Concrete is the most widely used material in construction industry, thanks to its relatively low cost combined with high compressive strength. However, concrete has to deal with durability issues, mainly caused by ingress of substances through interconnected pores, deteriorating the material.

A solution is the use of small additions of nanosilica [1]. These particles have a relatively small volume, making them an ideal candidate to fill small pores. On the other hand, they are characterized by a high surface area, which makes them highly reactive. The silica particles will react with $\mathrm{Ca}(\mathrm{OH})_{2}$, forming additional C-S-H. Both features influence the final properties of the concrete material.

Several researches have already been conducted on this topic, but no straightforward conclusion can be drawn regarding the evolution in mechanical properties. A study by $\mathrm{Yu}$ et al. [2] showed that the replacement of cement by some percentages of nanosilica slurry increased both the flexural and the compressive strength. An optimum amount was found to be around $3.7 \%$ of binder weight. Moreover, the replacement of cement by this optimum amount showed a denser microstructure compared to a lower or larger amount of nanosilica added. Ltifi replaced cement by colloidal silica in cementitious mortars [3]. In this research, compressive tests at different ages were conducted on mortar samples with a replacement of cement with $3 \%$ nanosilica. However, at a curing age of 28 days the compressive strength was lowered compared to the reference samples, while for 14 days the strength was comparable. In this case, the effect on the mechanical properties could thus not clearly be determined.

This paper studies the replacement of cement by colloidal

\footnotetext{
^e-mail: gerlinde.lefever@vub.be
}

(nano)-silica in mortar mixtures. A characterization of the different mortar mixtures is done by measuring the workability, the flexural and compressive strength and the heat of hydration. Differences in the behaviour upon bending are identified by comparing acoustic emission data received while performing three-point-bending tests.

\section{Materials and methods}

\subsection{Materials}

Three different mortar mixtures were made: a reference mixture (without colloidal silica) and two mixtures containing respectively $0.5 \%$ and $1 \%$ of colloidal silica by mass of cement. The cement used was CEM I $52.5 \mathrm{~N}$ produced by Diamur, Belgium. It is a Portland cement and consists mainly of Portland clinker with a maximum of $5 \%$ of other additives. The type of colloidal silica used was Ludox ${ }^{\circledR}$ HS-40, delivered by GRACE ${ }^{\circledR}$, USA. This colloidal dispersion contains $40 \%$ of silica, with a nominal particle size of $12 \mathrm{~nm}$. In the table below, some important typical properties of the colloidal silica are summarized.

Table 1. Typical properties of Ludox ${ }^{\circledR}$ HS-40

\begin{tabular}{c|c} 
Density & $1.3 \mathrm{~g} / \mathrm{cm}^{3}$ \\
\hline $\mathrm{pH}\left(25^{\circ} \mathrm{C}\right)$ & $9.2-9.9$ \\
\hline Specific surface area & $198-258 \mathrm{~m}^{2} / \mathrm{g}$ \\
\hline Stabilizing counter ion & sodium
\end{tabular}

Also, a superplasticizer, being MasterGlenium 51 (conc. $35 \%$ ) from BASF, was used to increase the workability of the mortar. The composition of all mixtures can be found in table 2 . 
Table 2. Compositon of mortar mixtures

\begin{tabular}{c|c} 
Water-to-binder ratio & 0.35 \\
\hline Sand-to-binder ratio & 2 \\
\hline Superplasticizer-to-binder ratio & 0.004
\end{tabular}

\subsection{Sample preparation}

Nine small beam elements were cast per mixture, measuring $40 \mathrm{~mm} \times 40 \mathrm{~mm} \times 160 \mathrm{~mm}$. The standard mixing procedure was as following:

1. Mixing of dry powders for $60 \mathrm{~s}$;

2. Adding water and superplasticizer;

3. Mixing for $180 \mathrm{~s}$

When nanosilica was added to the mixture, the colloidal silica was first placed in an ultrasonic bath for $5 \mathrm{~min}$ to achieve better dispersion within the solution. This solution was then added after $30 \mathrm{~s}$ of mixing of the dry powders.

After one day of curing, the samples were demoulded and wrapped in plastic foil till the age of testing to maintain the humidity. The samples are kept at a constant temperature of $20 \pm 1^{\circ} \mathrm{C}$.

\subsection{Testing methods}

\subsubsection{Flow table test}

The consistency or 'flow' of the different mortars was tested by means of a flow table. The procedure followed to measure the flow was European standard EN 1015-3 [4]. The diameter in two perpendicular directions gave an indication on the flow of the mortar and is a helpful tool for comparison with other mixtures in terms of the workability.

\subsubsection{Ultrasonic testing}

At a curing age of three, seven and 28 days, three beams of each mixture were tested by means of ultrasonic testing. Ultrasound waves were sent through the material and the travel time for the wave to pass through the beam was measured. The velocity of the wave depended on the density, the E-modulus and the Poisson's ratio of the material, as equation 1 describes:

$$
v=\sqrt{\frac{E(1-v)}{\rho(1+v)(1-2 v)}}
$$

with $\mathrm{v}=$ velocity $[\mathrm{m} / \mathrm{s}], \mathrm{E}=\mathrm{E}$-modulus $[\mathrm{Pa}], \rho=$ density $\left[\mathrm{kg} / \mathrm{s}^{3}\right]$ and $v=$ Poisson's coefficient [/].

\subsubsection{Flexural and compressive strength}

Identical as for the ultrasonic testing, flexural and compressive strength tests were performed at three, seven and 28 days of curing on the same three samples. The flexural strength of the beam samples was determined by means of a three-point bending test, following ASTM C 348-14 [5]. A notch of $5 \mathrm{~mm}$ is made in the middle of the beams to be sure on the fracture position. Afterwards, the prisms are broken in half and can be used for compressive strength determination (ASTM C 349-14 [6]).

\subsubsection{Acoustic Emission}

Acoustic emission (AE) is a non-destructive testing technique, based on the fact that materials emit elastic (sound) waves when subjected to stress. In order to obtain $\mathrm{AE}$ information, four piezoelectric sensors were placed on the beams while performing the three-point-bending tests. The set-up is depicted in figure 1 .

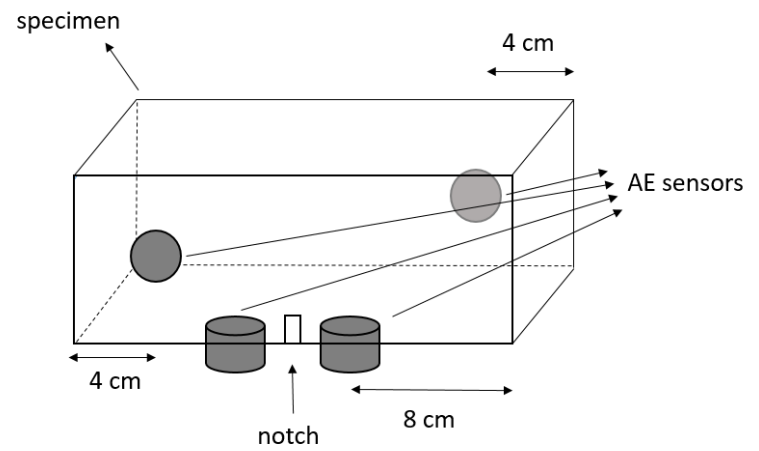

Figure 1. Lay-out of the acoustic emission sensors

A comparison between the $\mathrm{AE}$ data of the reference specimens and the specimens containing nanosilica was done in order to characterize differences in behaviour under flexural loading.

\subsubsection{Isothermal calorimetry}

Cement pastes were used to place in a TAM-AIR isothermal calorimeter. The three different mortar mixtures are thus reduced to three cement pastes. At a constant temperature of $20 \pm 1^{\circ} \mathrm{C}$, the generated thermal power was measured, which is the heat of hydration of the mortar samples. By means of this measuring technique, a delay or advance in generated heat could be determined and a comparison in released energy of the samples was performed.

\section{Results}

\subsection{Flow table test}

The results of the flow table test are listed in table 3 . From each mixture, two samples are tested. The workability decreases as more cement is replaced by colloidal silica, which means that the mixture becomes drier despite the fact that the water-to-binder ratio was constant. This is due to the very large surface area of the nanoparticles, retaining noteworthy amounts of water. 
Table 3. Results of the flow table test

\begin{tabular}{c|c|c|c} 
& Reference & $0.5 \mathrm{~m} \%$ HS40 & $1 \mathrm{~m} \%$ HS40 \\
\hline Flow [mm] & $165 \pm 4$ & $152 \pm 2$ & $121 \pm 3$
\end{tabular}

\subsection{Ultrasonic testing}

The ultrasonic wave velocity was measured and the density of the mortar beams was determined. Weighing the samples at different curing ages showed that the mass difference was below $1 \%$ so that the mass was considered to be constant. For each beam, the E-modulus was calculated assuming a Poisson's coefficient of 0.2. The evolution of the E-modulus is shown in figure 2.

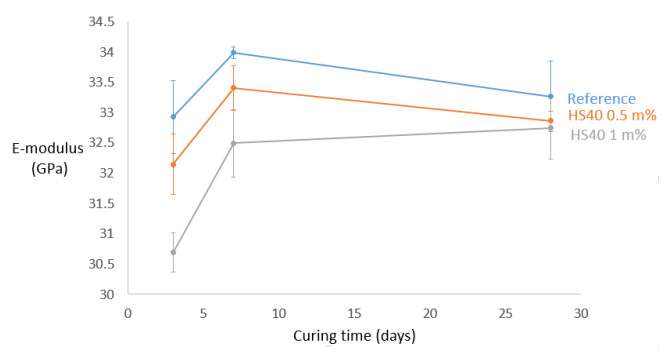

Figure 2. Evolution of the E-modulus

The E-modulus decreases for an increased amount of colloidal silica, which means that the material becomes less stiff due to the action of the HS40. However, for a longer curing time the error bars are overlapping, which means that after 28 days it can be said that the E-modulus is approximately the same for all mixtures.

It should be noted that we expected that the E-modulus would increase over time, as the hydration of the cement particles continued. However, for the reference samples and the samples with $0.5 \%$ of HS40, the E-modulus decreases slightly from seven to 28 days. The reason is still unclear, but it is possibly due to the variation on the results, as the ultrasonic wave velocity is highly dependent on the pressure that is manually applied to the sensors.

\subsection{Flexural and compressive strength}

The strength results of the three-point-bending and compression tests are depicted in figure 3 and 4 respectively. Three samples were tested to obtain the flexural strength, whereas the remaining halves (six per mixture) are used for compressive testing. Both flexural and compressive strength decrease when more cement is replaced by nanosilica. This can be due to the very poor workability of the mortar: the samples containing silica are more difficult to cast and compact, which causes the presence of a high amount of air bubbles and porosity at the top, negatively influencing the mechanical properties and increasing the varation on the results.

\subsection{Acoustic emission}

From the previous section no large differences between the flexural strength of the reference samples and the ones containing nanosilica were found. A deeper investigation on the behaviour in flexion will now be done by means of AE data.

Figure 5 shows the accumultative AE activity versus the load of a representative specimen of each mixture. The load is shown as a variation from zero to one, one being the maximum load or flexural strength. A significant difference in AE activity can be seen at the moment of reaching the peak load. For the reference samples, only a small proportion of hits occured at this moment, while for the other mixtures this proportion is quite high.

A closer look was made on the characteristics of the waveforms at the moment of fracture. Table 4 summarizes the absolute energy at fracture and the average duration of a hit at this moment. The energy is two orders of magnitude higher for the reference compared to the nanosilica samples and the average duration of a hit is three to four times as high. These results can be explained as following: the low amount of $\mathrm{AE}$ hits at fracture together with the longer duration for the reference samples indicates that large cracks are formed. This can also be confirmed by the high energy that is released during fracture. For the specimens containing nanosilica, smaller cracks are induced, but in a large amount. This explains the high amount of hits at the moment of fracture and the shorter duration of the hits. A similar study was conducted by Ioannou et al. [7]. Their study covered a comparison of mortars, with and without nanosilica, and the acoustic activity was monitored during three-point-bending tests. They concluded that the specimens containing nanosilica exhibit more shear fracture as compared with a reference mortar.

Table 4. Characteristics of the waveforms at fracture

\begin{tabular}{c|c|c|c} 
& Reference & $0.5 \mathrm{~m} \% \mathrm{nS}$ & $1 \mathrm{~m} \% \mathrm{nS}$ \\
\hline AE energy [attoJ] & $1.21 * 10^{6}$ & $1.77 * 10^{4}$ & $5.20 * 10^{4}$ \\
Duration $[\mu s]$ & 779 & 168 & 210 \\
Load drop $[\mathrm{kN}]$ & 1887.2 & 1450.3 & 1545.5
\end{tabular}

Finally, a different post-peak behaviour is seen for the nanosilica specimens compared to the reference: in figure 5 the load drop is smaller when nanosilica is present in the mixtures. The exact values are presented in table 4 .

All these results indicate that when cement is replaced by nanosilica, the specimens show a more ductile behaviour.

\subsection{Isothermal calorimetry}

The normalized heat flow [W/g] over time is received for the three different mixtures and a zoom on the first $30 \mathrm{~min}-$ utes is represented in figure 6 . It can be seen that when more nanosilica is present in the mixture, the peak in released energy occurs earlier compared to the reference, which means a faster hydration in nanosilica systems. This can be confirmed by literature: the nanosilica particles act as nucleation sites for the hydration reaction, increasing the rate of hydration. 


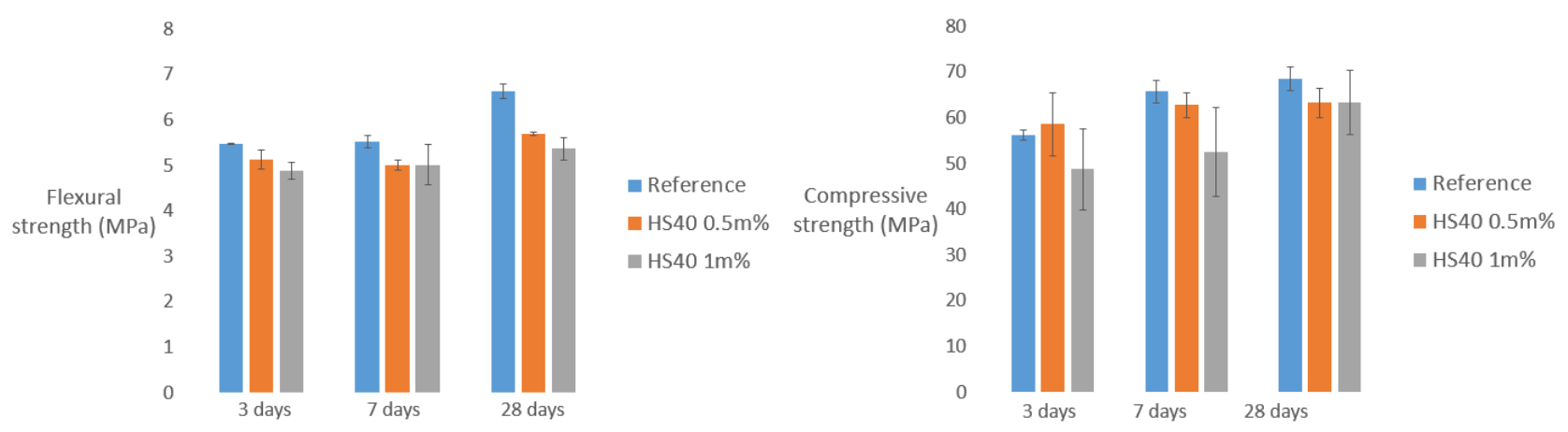

Figure 3. Evolution of the flexural strength

Figure 4. Evolution of the compressive strength

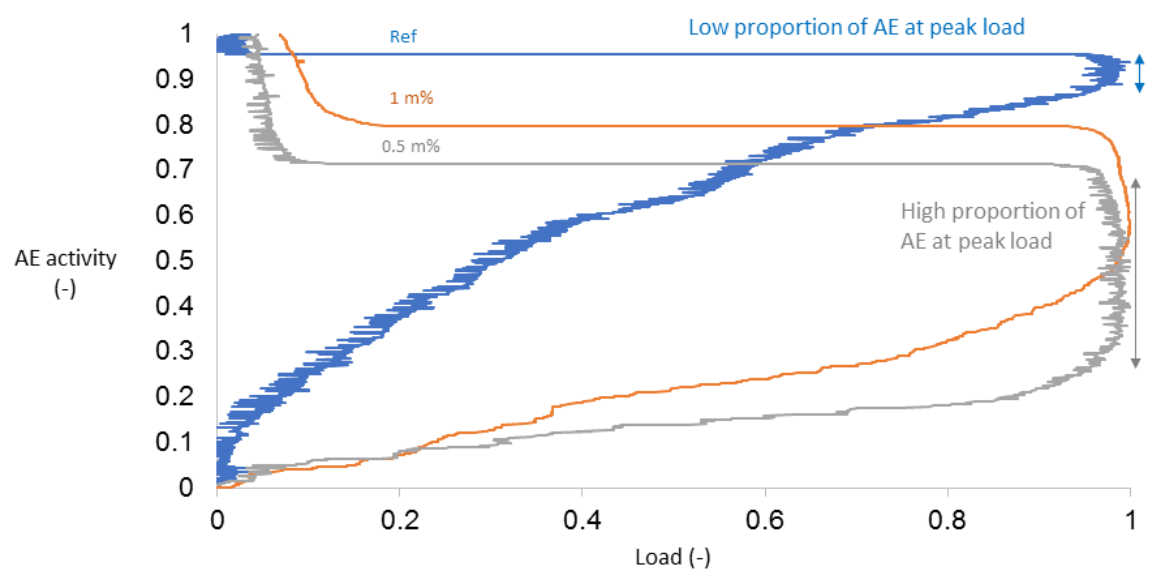

Figure 5. Evolution of the E-modulus

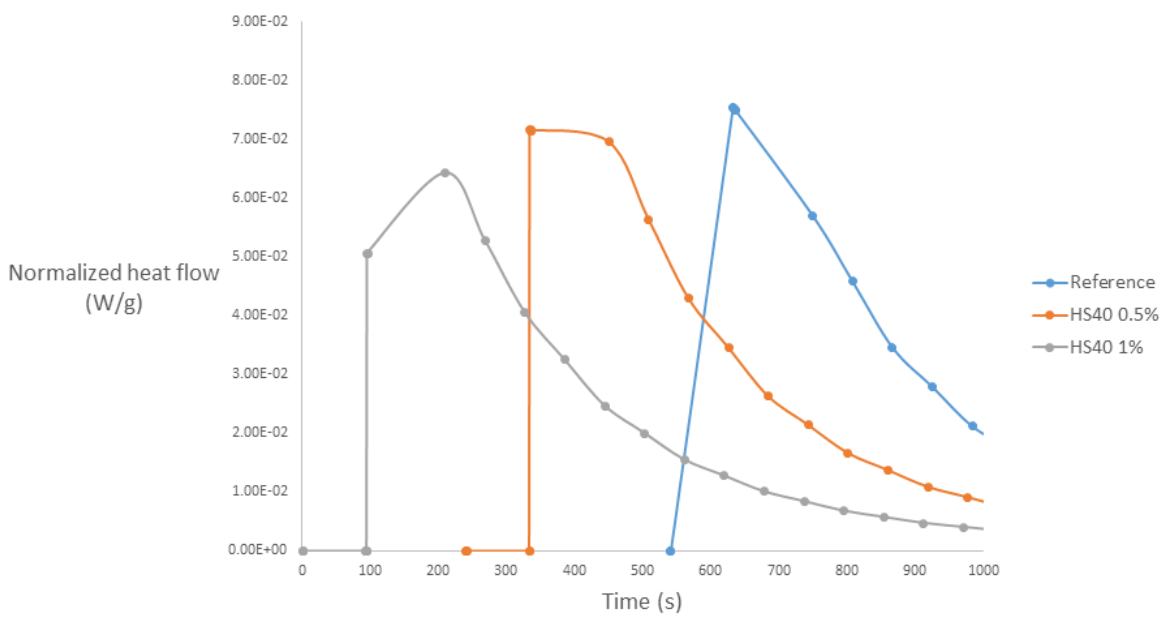

Figure 6. Normalized heat flow over time of the three mortar mixtures

\section{Conclusion and further research}

Although only small additions of cement were replaced by nanosilica, the influence on the mortar properties is not negligible. The two most important differences were seen in the workability and the behaviour in bending. The workability of the mortar decreases largely when more nanosilica is present in the mixtures, this due to the high surface area of the particles. On the other hand, it was seen that the beam specimens with nanosilica show a more ductile behaviour under three-point bending, which is confirmed by several characteristics of the $\mathrm{AE}$ waveforms at fracture.

However the difference is less pronounced for the results of E-modulus and compressive strength, it was seen that an overal decrease in these properties was obtained when silica is added. This is of course not desired and can also 
not be supported by literature.

Further research is needed to check whether there is a specific reason for this decrease in mechanical properties. The workability of the mortar and the dispersion of the particles plays an important role. One of the ideas is to keep the workability of mixtures with nanosilica constant by adjusting the amount of superplasticizer or to decrease the amount of superplasticizer in the reference mortar. In this way, a comparison with a new reference can be done and it can be assured that all samples are compacted and cast in an ideal, identical way.

This research is granted by the Research foundation - Flanders (FWO), grant number G.0A28.16. The authors wish to express their gratitude for this support.

\section{References}

[1] L. Singh, S. Karade, S. Bhattacharyya, M. Yousuf, S. Ahalawat, Construction and Building Materials 47,
1069 (2013)

[2] R. Yu, P. Spiesz, H. Brouwers, Construction and Building Materials 65, 140 (2014)

[3] A. Guefrech, P. Mounanga, A. Khelidj et al., Procedia Engineering 10, 900 (2011)

[4] EN 1015-3, European standard, Comité Européen de normalisation (CEN), Brussels, BE (1999)

[5] ASTM C 348-02, Standard, ASTM International, West Conshohocken, PA 19428-2959, United States (2002)

[6] ASTM C 349-02, Standard, ASTM International, West Conshohocken, PA 19428-2959, United States (2002)

[7] C. Ioannou, A. Iliadou, D. Aggelis, N. Barkoula, T. Matikas, Emerging Technologies in NonDestructive Testing V pp. 333-339 (2012) 